\title{
Renal Replacement Therapy in Pregnancy-related Acute Kidney Injury: Getting the Timing Right
}

\author{
Valentine A Lobo
}

\begin{abstract}
Recent trials have failed to show a survival benefit from the early initiation of dialytic therapies in acute kidney injury (AKI), but the problem has not been studied in pregnancy-related AKI. While the KDIGO criteria have not been validated in pregnancy-related acute kidney injury (PRAKI), additionally both fetal and maternal outcomes require to be studied. The short observational study by Banerjee et al. contains some interesting observations.

Keywords: Fetal loss, Pregnancy-related acute kidney injury, Renal replacement therapy.

Indian Journal of Critical Care Medicine (2020): 10.5005/jp-journals-10071-23540
\end{abstract}

Broad consensus exists that patients with acute kidney injury who have hyperkalemia, metabolic acidosis, volume overload, blood urea nitrogen $>100 \mathrm{mg} \%$, pericarditis, or encephalopathy need renal replacement therapy. ${ }^{1}$ All of these are now regarded as complications of AKI, and several trials have focused on achieving homeostasis by an earlier initiation of RRT at KDIGO stages 2 or 3 without complications. Initiation of RRT in the AKIKI STAART $\mathrm{AKI}^{2}$ and IDEAL ICU studies was considered to be delayed if the above complications were present. These three recent multicenter randomized controlled trials accounting for a total of 4,035 patients in either early or delayed strategies have shown no difference in the primary outcomes of 60- and 90-day mortalities, but revealed that $38-49 \%$ of patients randomized to a delayed strategy never received RRT. Additionally, the early strategy was associated with a three times higher risk of catheter-related bloodstream infection, ${ }^{3}$ hypotensive episodes, ${ }^{4}$ longer duration of RRT or dialysis dependence, ${ }^{3}$ and hypophosphatemia. ${ }^{4}$ These studies and an earlier RCT from India ${ }^{5}$ appear to establish an absence of benefit of an early initiation of RRT strategy and a signal of harm, although the study from KEM, Mumbai, was not carried out in an ICU but a ward where the patient and illness profile are very different. Importantly, $80 \%$ of the patients in AKIKI and all those in IDEAL-ICU had sepsis, while the population in STAART AKI was large enough and heterogeneous enough to allow for a subgroup analysis. The majority of evidence therefore appears to be stacked up against an early start.

Now Banerjee et al. ${ }^{6}$ in this issue of the journal presented an evidence that fetal outcomes may be improved with the early initiation of RRT in pregnancy-related acute kidney injury (PRAKI). There are multiple important implications of this study, which differ from the multiple high-quality trials. In the first place, the AKIN and KDIGO criteria for the classification of AKI, universally used in all the above trials, have not been validated in PRAKI. This group of patients may therefore behave differently and have special requirements, which have not been studied and need to be elucidated. Indeed, it is common for many studies to exclude pregnant patients from initial studies. Macedo and Mehta ${ }^{7}$ have suggested that the kidney possesses a finite capacity to support other organ systems and RRT should be viewed as a support to meet a supply-demand mismatch that exists in AKI. This problem
Department of Nephrology, King Edward Memorial Hospital, Pune, Maharashtra, India

Corresponding Author: Valentine A Lobo, Department of Nephrology, King Edward Memorial Hospital, Pune, Maharashtra, India, Phone: +919049232489, e-mail: valentinelobo702@gmail.com

How to cite this article: Lobo VA. Renal Replacement Therapy in Pregnancy-related Acute Kidney Injury: Getting the Timing Right. Indian J Crit Care Med 2020;24(8):624-625.

Source of support: Nil

Conflict of interest: None

may be particularly evident in PRAKI as all systems including the renal are already working at the maximum capacity of their functional reserve. ${ }^{6,8}$ Thus, any compromise may result in an inability to accommodate an increased metabolic and volume load. In fact, stable chronic patients on maintenance dialysis (MHD) require an increased dose of dialysis (20 hours per week) to successfully carry a pregnancy to term. ${ }^{9}$ The only study to show a benefit of early initiation, the ELAIN trial ${ }^{10}$ was a singlecenter study mainly in cardiac surgery patients, suggesting that different patient populations may have different needs and outcomes.

The second important message of this study is that it focuses on an important subclass of patients in India where $25 \%$ of critical care AKI patients requiring RRT may be pregnant women ${ }^{6}$ as opposed to developing countries where this subgroup is smaller.

The third unique aspect of PRAKI in general and in this study is that both maternal and fetal outcomes need to be measured. Because a large number of PRAKI are expected to improve with delivery, the impact of dialysis upon maternal rather than fetal outcomes is assessed. In this study, the authors show that maternal outcomes were not impacted, in line with the results of the other RCTs in adults, but a significantly better fetal outcome was observed in patients who received an early RRT.

This study has the above mentioned strengths, but also has several limitations. It is a single-center study with a small number of patients, an expected result as improved obstetric care has 
decreased the number of PRAKI. It may therefore contain a significant alpha error and, in the light of contrasting evidence from very high-quality multicenter studies, clearly needs validation in a multicenter randomized trial. Single-center studies have frequently been noticed to inflate an effect even in randomized trials as seen in the ELAIN study, which actually compared very early (KDIGO stage 2) with early (stage 3) AKI, and interestingly, $75 \%$ of the stage 3 patients had complications, such as pulmonary edema. With the demonstration that a significant number of patients may actually recover from AKI without RRT, given time, the impact of such a delayed strategy on fetal outcomes needs to be studied in an RCT with well-defined criteria. Another limitation of this study was that the initiation of RRT was driven by the preference of the primary care physician. This, however, may reflect the widespread practice where despite the evidence from trials, clinicians frequently take a more arbitrary decision on initiation, ${ }^{11}$ especially to control volume overload in anuric patients with a large mandatory projected intake in the ICU. The need for a randomized trial is again underlined by the fact that the two groups differed very significantly in blood pressure, anemia, sepsis, oliguria urea, and creatinine. It appeared that the early initiation group were clearly sicker and would have definitely had a worse outcome if the RRT had been delayed, another similarity with the ELAIN trial. ${ }^{11}$ Finally, the entire cohort was extremely heterogeneous with the etiology of AKI, including sepsis, malaria, and snake bite, which while characteristic of AKI in a developing country do not strictly merit consideration as a PRAKI. The results of this study therefore may not be applicable to a more classic cohort of PRAKI.

In conclusion, two important questions therefore still confront the community of intensivists, nephrologists, and obstetricians in India. They need to validate the KDIGO criteria of AKI in PRAKI, and a randomized multicenter study of timing of RRT in PRAKI that evaluates both maternal and fetal outcomes is urgently needed.

\section{REFERENCES}

1. Mehta RL. Renal-replacement therapy in the critically ill-does timing matter? N Engl J Med 2016;375(2):175-176. DOI: 10.1056/ NEJMe1606125.
2. The STARRT-AKI Investigators, for the Canadian Critical Care Trials Group, The Australian and New Zealand Intensive Care Society Clinical Trials Group, The United Kingdom Critical Care Research Group, the Canadian Nephrology Trials Network, and The Irish Critical Care Trials Group, Bagshaw SM, Wald R, Adhikari NKJ, Bellomo R, da Costa BR, et al. Timing of initiation of renal-replacement therapy in acute kidney injury. N Engl J Med 2020;383(3):240-251.

3. Gaudry S, Hajage D, Schortgen F, Martin-Lefevre L, Pons B, Boulet E, et al. Frederique schortgen et al for the AKIKI study group initiation strategies for renal-replacement therapy in the intensive care unit. N Engl J Med 2016;375(2):122-133. DOI: 10.1056/NEJMoa1603017.

4. Barbar SD, Clere-JehI R, Bourredjem $A$, Hernu R, Montini $F$, Bruyère $R$, et al. Timing of renal-replacement therapy in patients with acute kidney injury and sepsis. N Engl J Med 2018;379(15):1431-1442. DOI: 10.1056/NEJMoa1803213.

5. Jamale TE, Hase NK, Kulkarni M, Pradeep KJ, Keskar V, Jawale S, et al. Earlier-start vs usual-start dialysis in patients with communityacquired acute kidney injury: a randomized controlled trial. Am J Kidney Dis 2103;62(6):1116-1121. DOI: 10.1053/j.ajkd.2013.06.012.

6. Banerjee A, et al. Comparison of standard conservative treatment and early initiation of renal replacement therapy in pregnancy related acute kidney injury (PRAKI): a single centre prospective study. Ind J Crit Care Med 2020;24(8):688-694.

7. Macedo E, Mehta RL. When should renal replacement therapy be initiated for acute kidney injury? Semin Dial 2011;24(2):132-137. DOI: 10.1111/j.1525-139X.2011.00838.x.

8. Hladunewich MA, Hou S, Odutayo A, Cornelis T, Pierratos A, Goldstein $M$. Intensive hemodialysis associates with improved pregnancy outcomes: a canadian and United States cohort comparison. J Am Soc Nephrol 2014;25(5):1103-1109. DOI: 10.1681/ASN.2013080825.

9. Bramham K, Nelson-Piercy C. Pregnancy and renal physiology. In: Turner NN, Lameire N, Goldsmith DJ, Winearls CG, Himmelfarb J, Remuzzi G, ed. Oxford textbook of Clinical Nephrology. 4th ed., 2016. pp. 2190-2198.

10. Zarbock A, Kellum JA, Schmidt C, Aken HV, Wempe C, Pavenstädt H, et al. Effect of early vs delayed initiation of renal replacement therapy on mortality in critically ill patients with acute kidney injury the ELAIN randomized clinical trial. JAMA 2016;315(20):2190-2199. DOI: 10.1001/ jama.2016.5828.

11. Gaudry S, Quenot JP, Hertig A, Barbar SD, Hajage D, Ricard JD, et al. Timing of renal replacement therapy for severe acute kidney injury in critically ill patients. Am J Respir Crit Care Med 2019;199(9):1066-1075. DOI: $10.1164 / \mathrm{rccm} .201810-1906 C P$. 